\title{
Systemic Nocardiosis as Unusual Clinical Onset of Late Advanced HIV Infection: A Case Report
}

\author{
Santoro Carmen Rita ${ }^{1}$, Brindicci Gaetano ${ }^{2,}$,, Mazzola Michele ${ }^{1}$, Giannelli Anna ${ }^{1}$, \\ Infante Giovanni $^{1}$, Fiorella Carmen Silvia ${ }^{1}$, Pappalettera Antonio ${ }^{1}$, Capodivento Saverio ${ }^{3}$, \\ Cascarano Marina ${ }^{3}$, Quinto Nicola ${ }^{3}$, Losappio Ruggero ${ }^{1}$, Laura Monno ${ }^{2}$ \\ ${ }^{1}$ Operative Unit of Infectious Diseases, Ospedale Civile Vittorio Emanuele II, Bisceglie, Italy \\ ${ }^{2}$ Operative Unit of Infectious Diseases, AOU Consorziale Policlinico, Bari, Italy \\ ${ }^{3}$ Operative Unit of Radiodiagnostic, Ospedale Civile Vittorio Emanuele II, Bisceglie, Italy
}

Email address:

gaetanobrindicci@gmail.com (B. Gaetano)

${ }^{*}$ Corresponding author

\section{To cite this article:}

Santoro Carmen Rita, Brindicci Gaetano, Mazzola Michele, Giannelli Anna, Infante Giovanni, Fiorella Carmen Silvia, Pappalettera Antonio, Capodivento Saverio, Cascarano Marina, Quinto Nicola, Losappio Ruggero. Systemic Nocardiosis as Unusual Clinical Onset of Late Advanced HIV Infection: A Case Report. International Journal of Infectious Diseases and Therapy. Vol. 3, No. 3, 2018 , pp. $62-65$. doi: $10.11648 /$ j.ijidt.20180303.13

Received: August 3, 2018; Accepted: September 12, 2018; Published: October 25, 2018

\begin{abstract}
The genus Nocardia includes aerobic actinomycetes causing disease in both humans and animals. Nocardiosis is a rare bacterial infection, but the incidence of human disease has increased in the recent years in association with an increasing population of immune-compromised hosts and the improved methods for detection and identification of Nocardia spp. in the clinical laboratory. All those situations of immune-system compromise are conditions associated with increased risk, especially when cell-mediated immunity is involved. The prognosis depends both upon the immediacy of therapy initiation, concomitant disorders and the immune status of the patient. Recently cases of multi-drug resistance nocardiosis and the epidemiology and drug susceptibility of less common Nocardia species have been described. In HIV positive patients pulmonary nocardiosis, the most common clinical presentation, has a low reported prevalence of $0.3 \%$, and it is not considered an AIDS-defining disease. The difficulties in isolation, the non-specific clinical and radiologic presentation, and the widespread use of cotrimoxazole (TMP/SMX) prophylaxis could be responsible for this low prevalence. In this paper, it is described a rare and fatal case of systemic nocardiosis arising with chest pain in a patient with a severe immune deficiency, unaware of his HIV positivity. Systemic nocardiosis is generally caused by $N$. asteroids and the prognosis is good, but in patients with AIDS, mortality in disseminated forms with central nervous system involvement is more than $50 \%$.
\end{abstract}

Keywords: Disseminated Nocardiosis, AIDS, Late Advanced Naive

\section{Introduction}

Nocardia is a genus of aerobic Actinomycetes responsible for localized or disseminated disease in animals and humans. The genus is named after Edmond Nocard, who, in 1888, described an aerobic filamentous Gram-positive, weakly acid-fast bacteria isolated in cattle. Eppinger reported the first human case of nocardiosis in 1890. Nocardia are widespread in the environment and are found in soil, vegetation, other organic matter, and also in fresh and salt water [1].
Nocardiosis is a rare bacterial infection, but the incidence of human disease has increased in the recent years in association with an increasing population of immunecompromised hosts and the improved methods for detection and identification of Nocardia spp. in the clinical laboratory $[2,3]$. Nocardia infections can also be found in immunecompetent individuals. It occurs at all ages and in all races with a male:female ratio of $3: 1$ (which is probably related to an exposure discrepancy with an underlying immune compromise in $60 \%$ of cases). The bacteria usually enters the body through the lungs, while more rarely the infection 
occurs through lesions on the skin. Dissemination via the bloodstream then determines the involvement of the lungs or other organs (brain, skin, kidneys, liver, spleen, adrenal glands), [1]. Cell-mediated immunity is important for control of the infection and the organisms survive inside macrophages for long periods of time [4].

The taxonomy of Nocardia as a species has been challenging because numerous revisions have been made with the identification of more than 85 species [5]. The most important species that can infect humans and determine both pulmonary and systemic involvement are Nocardia asteroides, $N$. farcinica, $N$. nova, and $N$. abscessus. $N$. brasiliensis is the most important species associated with cutaneous lesions and lymphadenitis [6].

Conditions associated with increased risk are: bone marrow or solid-organ transplantation, immune-suppressive therapy or long-term steroid therapy, human immunedeficiency virus (HIV)/acquired immune-deficiency syndrome (AIDS) with CD4 $<50$ cells/mm3, malignancies, hypogammaglobulinemia and all situations of immunesystem compromise, especially when cell-mediated immunity is involved [7]. The prognosis depends both upon the immediacy of therapy initiation, concomitant disorders and the immune status of the patient [8].

Furthermore, cases of multi-drug resistance nocardiosis [9, 10] and the epidemiology and drug susceptibility of less common Nocardia species have recently been described [11, 12]. In HIV- positive patients pulmonary nocardiosis, the most common clinical presentation, has a low reported prevalence of $0.3 \%$ [13], and it is not considered an AIDSdefining disease. The difficulties in isolation, the nonspecific clinical and radiologic presentation, and the widespread use of cotrimoxazole (TMP/SMX) prophylaxis could be responsible for this low prevalence [13].

In this work it is described an unusual case of severe disseminated Nocardiosis in a late, advanced HIV-positive patient.

\section{Case Report}

This case concerns an Italian man of 56 living in South Italy (Andria) who had been married for about 25 years, fathering two children. The patient reported that he worked as a butcher and had been always in good health, except for a form of seborrheic dermatitis, which had started in 2006 . He was neither a smoker nor an alcohol or drugs user. In the January 2016 the patient referred a mono-metameric zoster infection of the right hemithorax, which was treated at home with acyclovir (800 mg tablets five times a day for seven days). On re-occurrence of a chest pain in July 2016 he underwent a chest X-ray with detection of cavitation (lower right lobe) and went into a Thoracic Surgery Department in a North Italian Centre (where he had relatives). The next chest TC showed a cavitated nodule of approximately $5 \mathrm{~cm}$ (in the lower right lobe). Blood tests showed a mild anemia ( $\mathrm{Hb} 10.6$ $\mathrm{g} / \mathrm{dl})$ and an increase of the erythrosedimentation rate (53 $\mathrm{mm} / \mathrm{h}$ ). The tests for the detection of Mycobacterium tuberculosis sputum and Quantiferon TB Gold resulted negative as did the neoplastic markers.

The patient also performed a cerebral CT in the same clinic department, which showed cystic site training vermian causing displacement of the fourth cerebral ventricle. An MRI of the brain was then performed, confirming the presence of the lesion, although it excluded the metastatic nature of the lesion. On the other hand, the suspicion of a brain neoplasm was proposed (hemangioblastoma). Lastly the patient underwent FDG PET/BT tests, revealing a hypermetabolic lesion on both lungs as well as on the right and left adrenal glands. Given that a pulmonary neoplasm was suspected, the patient underwent a bronchoscopy with a biopsy of the nodule, showing the following: "....a necrotizing granulomatous reaction, absence of cancer cells, acid-fast bacilli alcohol, hyphae and/or fungal spores".

Nevertheless, the patient was subjected to a right-lower pulmonary lobectomy. The extemporaneous histological examination was performed on one of the pulmonary lesions with evidence of "...the presence of cellular atypia compatible with non-small-cell lung cancer".

Ten days after the patient was discharged in late September, the final pathology report was drafted, pointing to the presence of filamentous micro-organisms, which were visible thanks to Grocott colouring and related "to Nocardia in the first line".

For this reason a specialist visit was recommended and the patient turned up at our department on October $15^{\text {th }}, 2016$, immediately undergoing a third-generation HIV ELISA test, which resulted positive. Thus the patient underwent serology testing for HBV, HCV, CMV, EBV, Toxoplasmosis, HSV, syphilis. On October 15th, the patient was hospitalized for a persistent abdominal pain, the appearance of fever and in order to undergo therapy. On admission a physical exam was performed without evidence of abnormalities.

The HIV-RNA level was 43,000 copies $/ \mathrm{ml}$, and an advanced stage of immunodeficiency with a total number of CD4 lymphocytes of 43 cells $/ \mathrm{mm}^{3}(6 \%)$ was reported. Blood-chemistry tests confirm a microcytic anemia and an increase in ESR without further anomalies (auto-immunity exams, neoplastic negative markers).

During hospitalization a TC total body was performed, showing multiple left lung nodules (the largest of which being $15 \mathrm{~mm}$ ). In addition, the CT scan signaled the presence of bilateral adrenal nodules (35 and $12 \mathrm{~mm}$ ) (figure 1).

On October $25^{\text {th }}, 2016$, an anti-retroviral therapy of tenofovir, emtricitabine and dolutegravir in conjunction with an antibiotic intravenous therapy using amikacina $(500 \mathrm{mg}, 3$ times daily) and cotrimoxazole ( $80 / 400 \mathrm{mg}$, three times daily) was administered.

The patient was hospitalized for about a month, after which he decided to self-adjust and voluntarily suspend antibiotic therapy (November $26^{\text {th }}$ ) by continuing only with cotrimoxazole and azithromycin tablets as prescribed for prophylaxis of opportunistic infections as well as for antiretroviral therapy. In mid-December, owing to the recurrence of fever and the persistence of hemithorax pain, 
the patient would be re-admitted and resumed intravenous infusion of antibiotic therapy.
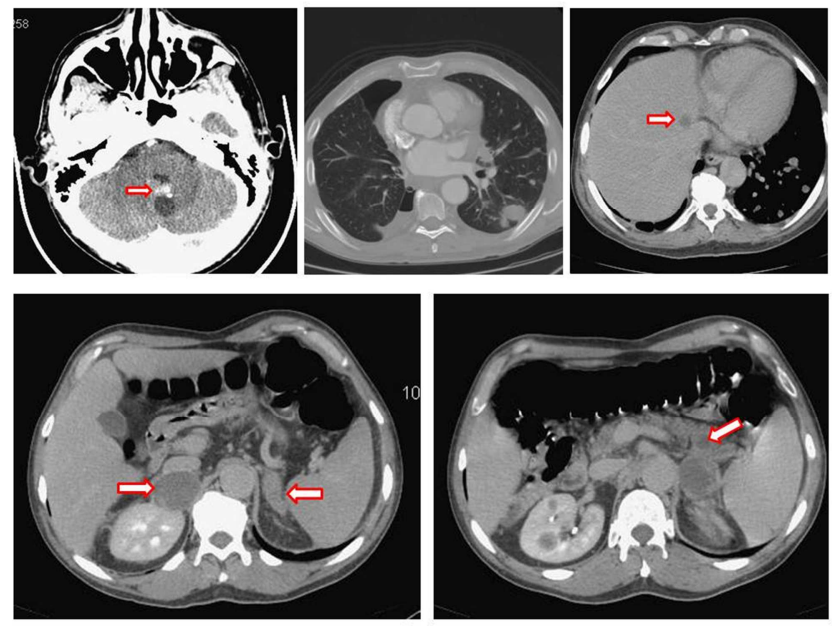

Figure 1. The first total body TC showed a cavitated nodule of $5 \mathrm{~cm}$ in the right-lower lobe, multiple contra-lateral nodules and bilateral adrenal nodules (35 and $12 \mathrm{~mm}$ ).

Total-body TC performed in December $16^{\text {th }} 2016$ showed that the radiological signs had worsened (figure 2) with unchanged persistence of the brain lesion and those in the lung and the right adrenal gland, not to mention the appearance of new lesions in the liver, pancreas and kidneys.
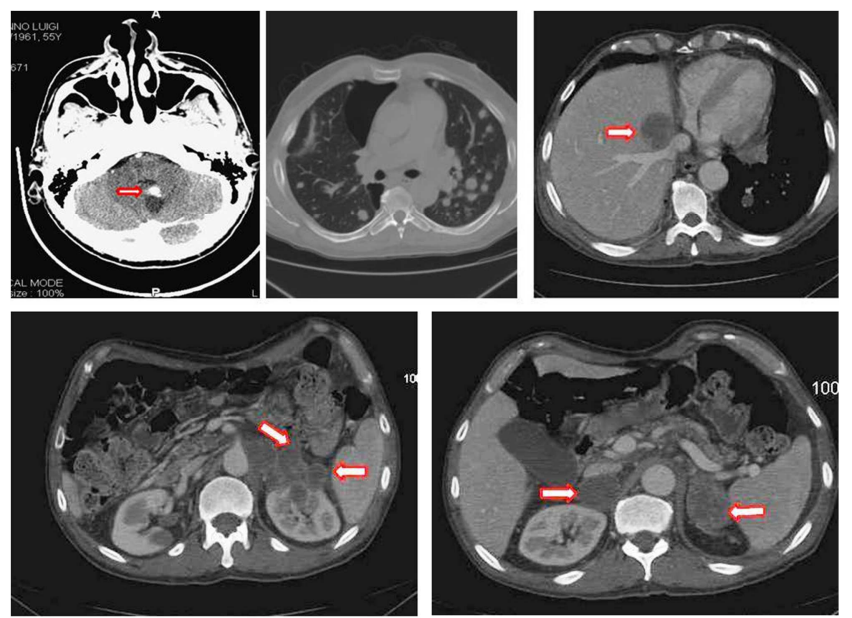

Figure 2. Total-body TC performed in December 2016 showed hat the radiological signs had worsened (unchanged persistence of the brain, lung $e$ right adrenal gland) and appearance of new lesions in the liver, pancreas and kidneys.

Imipenem (500 $\mathrm{mg}$ in 4 doses) was added to the ongoing therapy. After about 1 month of hospitalization, a new total body TC was performed, showing a reduction in the number and size of lung lesions as well as a reduction in the volume of pancreatic and cerebral lesions. On the contrary, the liver abscess and kidney lesions had increased on diameter.

The adrenal abscesses were unchanged. On February 2017 a new TC total body was performed, with evidence of further worsening of both lung, brain, liver and kidney lesions. The pancreatic lesion was the only unchanged event.
Clinically, one could note a gradual deterioration in the patient, who appeared to be worn out, asthenic, but also anorexic, an exitus occurring a few days later.

\section{Discussion}

Advances in the field of anti-retroviral therapy over the last 25 years have in fact obviously reduced the opportunistic diseases that still occur in advanced-naïve cases. Nocardiosis is a rare disease whose prevalence in recent decades has increased concurrently with the increase in immunosuppressed patients.

It is estimated that at least $50 \%$ of patients with an $N$. asteroides infection are immune-compromised [14] and approximately $4 \%$ of patients who die of AIDS have evidence of nocardiosis on autopsy [15].

Since the clinical and radiologic manifestations are non specific and the microbiological diagnosis is often difficult, it seems likely that, in some patients, pulmonary nocardiosis will be mistaken for other infections, such as tuberculosis, bacterial pneumonia, or malignancies. In the represented case the patient with a severely impaired cell-mediated immunity presented a serious case of disseminated nocardiosis. Although this infection is not among the AIDS-defining illnesses, there is no doubt that it must always be sought in the case of multiple locations. Conversely, HIV testing is a must if it is placed before the diagnosis of nocardiosis.

Systemic nocardiosis is generally caused by N. asteroids and the prognosis is good if antibiotic therapy is started promptly and aggressively. However, in patients with AIDS, mortality in disseminated forms with central nervous system involvement is more than $50 \%$. International guidelines provide for continuous intravenous treatment for at least 12 months in the most severe cases. The prognosis is also related to the initial immune-deficiency state. In this case there was a severe deficiency of cell-mediated immunity which, despite the effectiveness of anti-retroviral therapy leading to the non-detectability of the HIV virus within a month from the beginning, did not associate itself with an equally-rapid increase in CD4+ cells responsible for immune response. It is well known that the possibility of immunelogical recovery depends on several factors including the years of infection, the nadir of $\mathrm{CD} 4+$, the ratio of $\mathrm{CD} 4$ naive/CD4 memory, and also on comorbidities. It is not unreasonable to think that the patient had been HIV-positive for ten years (since being diagnosed for seborrheic dermatitis). In fact, seborrheic dermatitis is known to be one of the first clinical manifestations associated with infection. The fact that the patient decided to opt out from the therapy and hence to suspend for about 20 days definitely worsened the situation and made the prognosis less meaningful.

\section{Conclusion}

Nocardiosis is an acute, subacute, or chronic infectious disease that occurs in cutaneous, pulmonary, and disseminated forms. 
Most patients with disseminated nocardiosis have underlying immunocompromising disease or are receiving immunosuppressive therapy and the infection results from hematogenous dissemination, usually from a pulmonary focus.

The HIV test should be offered to all patients with nodules or cavitated lung lesions. In patients with HIV/AIDS who manifest symptoms of pulmonary infiltrates, Nocardia infections should be considered in the differential diagnosis. Increased awareness of the infection in patients with advanced disease (CD4+ cell counts less than $100 / \mu \mathrm{L}$ ), especially in the absence of pneumocystis jirovecii pneumonia prophylaxis with TMP/SMX, may facilitate earlier diagnosis and timely treatment of this infection, resulting in better survival.

\section{Acknowledgements}

The authors thank Dr. James Hart for the helpful review of the manuscript.

\section{Conflicts of Interest}

The authors declare that they have no competing interests.

\section{References}

[1] Fatahi-Bafghi M. Nocardiosis from 1888 to 2017. Microb Pathog. 114:369-384, 2018.

[2] Conville P.S., Brown-Elliott B.A., Smith T., Zelazny A.M. The Complexities of Nocardia Taxonomy and Identification. J Clin Microbiol. 56(1), 2017.

[3] Brown-Elliott B.A., Brown J.M., Conville P.S. and Wallace R.J. Jr. Clinical and laboratory features of the Nocardia spp. based on current molecular taxonomy. Clin Microbiol Rev 19: 259-282, 2006.

[4] Corti M.E., Villafafie Fioti M.F. Nocardiosis: a review. Int J Infect Dis 7: 243-250, 2003.
[5] Wilson J.W. Nocardiosis: Updates and Clinical Overview. Mayo Clin Proc. 87(4):403-407, 2012.

[6] Yu S., Wang J., Fang Q., Zhang J., Yan F. Specific clinical manifestations of Nocardia: A case report and literature review. Exp Ther Med. 12(4): 2021-2026, 2016.

[7] Peleg A.Y., Husain S., Qureshi Z.A. et al. Risk factors, clinical characteristics, and outcome of Nocardia infection in organ transplant recipients: a matched case-control study. Clin Infect Dis. 44:1307-1314, 2007.

[8] Kim Y.K., Sung H., Jung J. et al. Impact of immune status on the clinical characteristics and treatment outcomes of nocardiosis. Diagn Microbiol Infect Di. 85(4):482-7, 2016.

[9] Senard O. Blanot S. Jouvion G. et al. Fulminant Nocardiosis Due to a Multidrug-Resistant Isolate in a 12-Year-Old Immunocompetent Child. Pediatrics. 141(2). pii: e20163131. doi: 10.1542/peds.2016-3131, 2018.

[10] Cooper C.J., Said S., Popp M. et al. A complicated case of an immunocompetent patient with disseminated nocardiosis. Infect Dis Rep. 6(1):5327, 2014.

[11] Valdezate S., Garrido N., Carrasco G. et al. Epidemiology and susceptibility to antimicrobial agents of the main Nocardia species in Spain. J Antimicrob Chemother. 72(3):754-761, 2017.

[12] Haussaire D., Fournier P.E., Djiguiba K. et al. Nocardiosis in the south of France over a 10-years period, 2004-2014. Int J Infect Dis. 57:13-20, 2017.

[13] King A.S., Castro J.G., Dow G.C. Nocardia farcinica lung abscess presenting in the context of advanced HIV infection: spontaneous resolution in response to highly active antiretroviral therapy alone. Can J Infect Dis Med Microbiol. 20:103-106, 2009.

[14] Palmer D.L., Harvey R.L., Wheeler J.K. Diagnostic and therapeutic considerations in Nocardia asteroids infection. Medicine (Baltimore). 53:391-401, 1974.

[15] Lucas S.B., Hounnou A., Peacock C., Beaumel A., Kadio A., De Cock K.M. Nocardiosis in HIV-positive patients: an autopsy study in West Africa. Tuber Lung Dis. 75:301-307, 1994. 